OPEN

SUBJECT AREAS:

POLYMERS

CHEMICAL ENGINEERING

FLUIDICS

COMPOSITES

Received

5 June 2013

Accepted

20 September 2013

Published

23 October 2013

Correspondence and requests for materials should be addressed to

E.B. (edward@ariel. ac.il)

\title{
Electrically Controlled Membranes Exploiting Cassie-Wenzel Wetting Transitions
}

\author{
Edward Bormashenko ${ }^{1,2}$, Roman Pogreb1, Sagi Balter ${ }^{2,3}$ \& Doron Aurbach ${ }^{3}$
}

\begin{abstract}
${ }^{1}$ Ariel University, Physics Faculty, 40700, P.O.B. 3, Ariel, Israel, ${ }^{2}$ Ariel University, Department of Chemical \& Biotechnology Engineering, P.O.B. 3, 40700, Ariel, Israel, ${ }^{3}$ Department of Chemistry, Bar-llan University, 52900 Ramat-Gan, Israel.
\end{abstract}

We report electrically controlled membranes which become permeable when an electrical field is exerted on a droplet deposited on the membrane. Micro-porous polycarbonate membranes are obtained with the breath-figures assembly technique, using micro-scaled stainless steel gauzes as supports. The membranes demonstrate pronounced Cassie-Baxter wetting. Air cushions trapped by the droplet prevent water penetration through the membrane. We demonstrate two possibilities for controlling the permeability of the membrane, namely contact and non-contact scenarios. When an electrical field is exerted on a droplet deposited on the membrane, the triple-line is de-pinned and the wetting transition occurs in the non-contact scheme. Thus, the membrane becomes permeable. The contact scheme of the permeability control is based on the electrowetting phenomenon.

\footnotetext{
- lectrically controlled membranes attracted the attention of investigators in light of their applications for microfluidics, slow-release drug delivery, transport of proteins and $\mathrm{DNA}^{1-6}$. We propose a novel approach to the elaboration of electrically controlled membranes, namely exploiting Cassie-Wenzel transitions induced by imposing an electric field on a droplet deposited on a porous permeable polymer substrate. It is well-known that exerting an electric field may modify the wetting regime of a solid/liquid pair $^{7-11}$. This takes place in electrowetting experiments in which the surface tension at the solid/liquid interface is influenced by an electric field ${ }^{8-11}$. In the classical electrowetting scheme a droplet is located between two electrodes, one of which touches the droplet (see Fig. 1a) $)^{7}$. In the electrowetting-on-dielectric (EWOD) scheme of electrowetting a droplet contacts with a dielectric layer coating the electrode ${ }^{7,12-15}$. The EWOD scheme allows significant decreases in the voltage necessary for the control of wettability. In our paper we demonstrate the possibility of exploiting the EWOD technique for controlling the permeability of polymer membranes assembled on micrometrically scaled metallic gauzes serving as electrodes.

We also propose a non-contact scheme of controlling the permeability of membranes in which electrodes do not touch a droplet, as shown in Fig. 1b. A droplet deposited on the micro-porous permeable surface is deformed by an external electric field ${ }^{16-18}$, and its triple (three-phase) line is detached from the substrate. Thus, the CassieWenzel transition occurs ${ }^{19-22}$ and the liquid penetrates the membrane.
}

\section{Results}

Manufacturing membranes. The microscopically scaled membranes were prepared with the "breath-figures self-assembly" technique using stainless steel micro-porous gauzes as supports (as described in detail in the Methods section and Ref. 23). The SEM image of the gauze is presented in Fig. 2. The use of stainless steel microporous gauzes provided the water permeability of the membranes. Since the process of "breath-figures selfassembly" was reported by Widawski, Rawiso and Francois ${ }^{24}$, the theoretical and experimental activities in this field have been extended over the past decade ${ }^{25-29}$. Despite the fact that the physical mechanism of the patterning phenomenon is not clear to a full extent, the water-assisted self-assembly technique has already been successfully applied for manufacturing strictly ordered, closely packed micro- and nanoscale porous $2 \mathrm{D}$ structures ${ }^{26-27}$. Generally, the "breath-figures self-assembly" is related to the condensation of micro-scaled water droplets on the cooled surface of an evaporated polymer solution ${ }^{25,28}$. The droplets then sink into the solution, eventually forming the honeycomb pattern ${ }^{25-28}$. It was already demonstrated that polycarbonate (PC), which is an amorphous thermoplastic polymer, possessing high mechanical, electric and thermal properties (a glass transition temperature of about $147^{\circ} \mathrm{C}$ ), may be effectively used for breath-figures self-assembly $y^{29,30}$. 


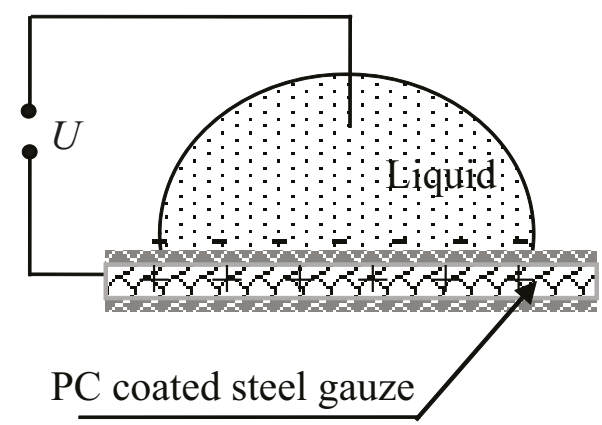

a

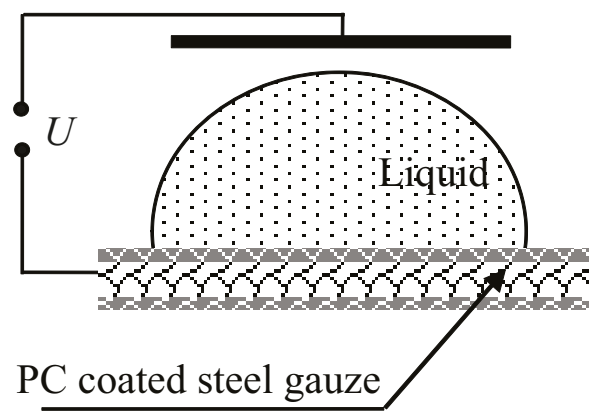

b

Figure $1 \mid$ (a) Traditional scheme of an electro-wetting experiment. $U$ is the voltage. The contact angle is changed due to formation of the double Helmholtz layer at the solid/liquid interface. The solid substrate in the PC coated gauze. (b) Non-contact scheme: the droplet deposited on the membrane is exposed to a uniform electric field.

PC membranes self-assembled with micro-porous stainless steel supports are depicted in Fig. 3. The average diameter of pores was about $5 \mu \mathrm{m}$.

$20 \mu \mathrm{l}$ distilled water droplets were deposited on the membranes. The membranes demonstrated pronounced Cassie-Baxter wetting ${ }^{22,31-34}$. The apparent contact angle was established as $125 \pm 1^{\circ}$, whereas the static (or "as placed", see Ref. 35) contact angle for the same solution deposited on atomically flat PC was $76 \pm 1^{\circ}$. The Cassie-Baxter wetting regime means that a droplet is supported partially by polymer (PC) and partially by air ${ }^{8,22,31-34}$. This makes the membrane impermeable to water.

Control of the permeability of membranes by electrowetting. We demonstrate the possibility to control the permeability of the membranes by exploiting the electrowetting effect ${ }^{7}$. When an aqueous electrolyte contacts a solid surface, a double electrical layer is formed ${ }^{7-11}$. The double layer works as a capacitor; thus the effective energy of the solid/liquid interface may be written as:

$$
\gamma_{S L}=\gamma_{S L}^{0}-\frac{1}{2} C U^{2}
$$

where $\gamma_{S L}^{0}$ represents the solid/liquid interface tension at zero voltage, $C$ is the capacitance per unit area of the substrate, and $U$ is the voltage, applied between electrodes as shown in Fig. 1a. Substitution of Exp. (1) into the Young Formula: $\cos \theta=\frac{\gamma_{S A}-\gamma_{S L}}{\gamma}$, yields for the

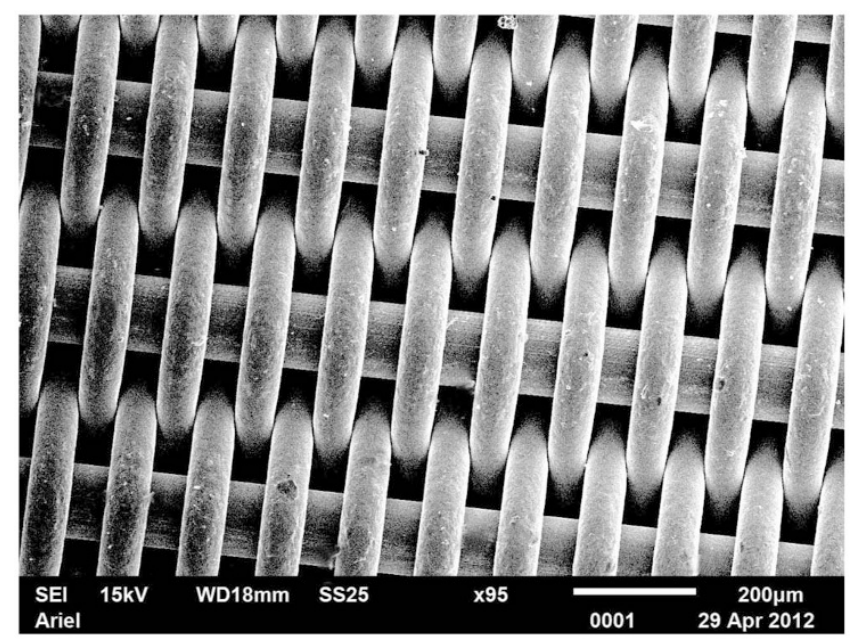

Figure 2 SEM image of the stainless steel wire gauzes used as supports for breath-figures self-assembly. The scale bar is $200 \mu \mathrm{m}$.

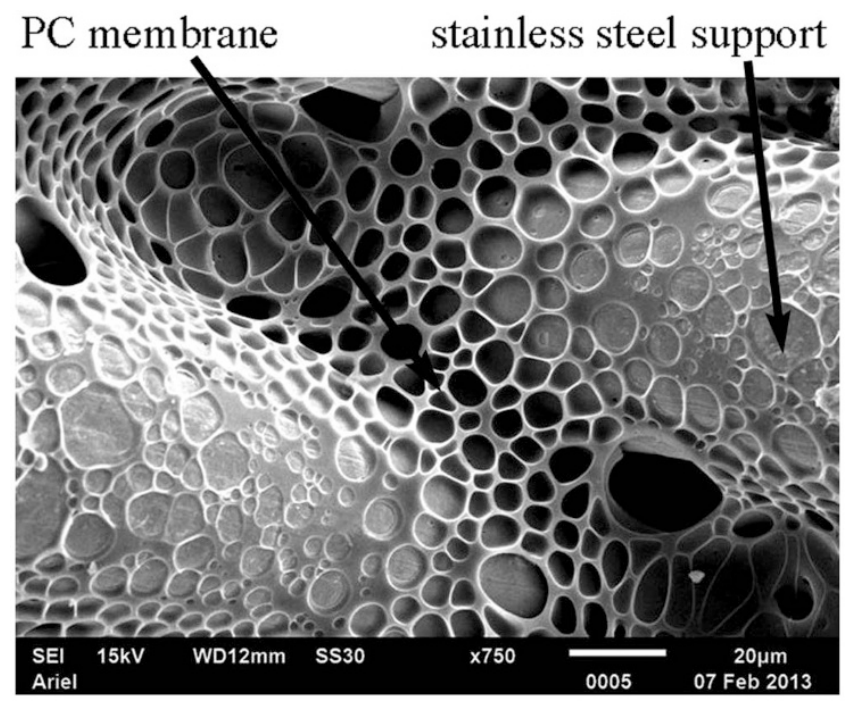

Figure 3 SEM image of the stainless steel gauzes coated with porous polycarbonate film with the breath-figures self-assembly. The scale bar is $20 \mu \mathrm{m}$.

electrowetting of a flat surface a contact angle $\theta_{e l}$ :

$$
\cos \theta_{e l}=\frac{\gamma_{S A}-\gamma_{S L}^{0}+\frac{C U^{2}}{2}}{\gamma}=\cos \theta_{Y}+\frac{C U^{2}}{2 \gamma} .
$$

Generalization of Exp. (2) for rough and chemically heterogeneous surfaces (and this is the case in our study) was reported in Ref. 11. When a droplet is deposited on PC-coated gauze as shown in Fig. 1a, we have a modification of the EWOD scheme with the gauze serving as an electrode. We established that voltages as high as $250 \mathrm{~V}$ are necessary for noticeable change of the apparent contact angle

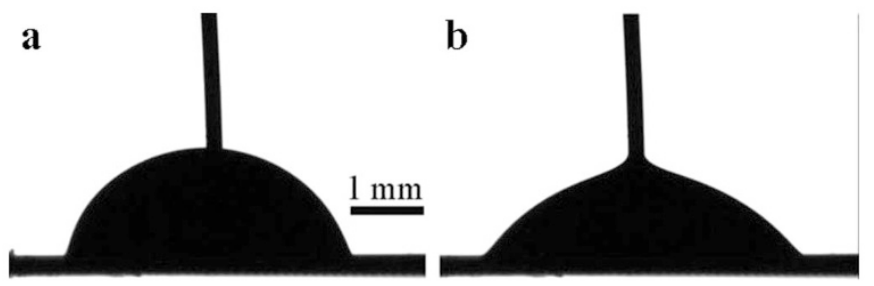

Figure $4 \mid$ Opening the membrane by electrowetting. A $20 \mu \mathrm{l}$ droplet deposited on the plasma treated PC-coated metal gauze: (a) $U=0 \mathrm{~V}$; (b) $U=30 \mathrm{~V}$. 


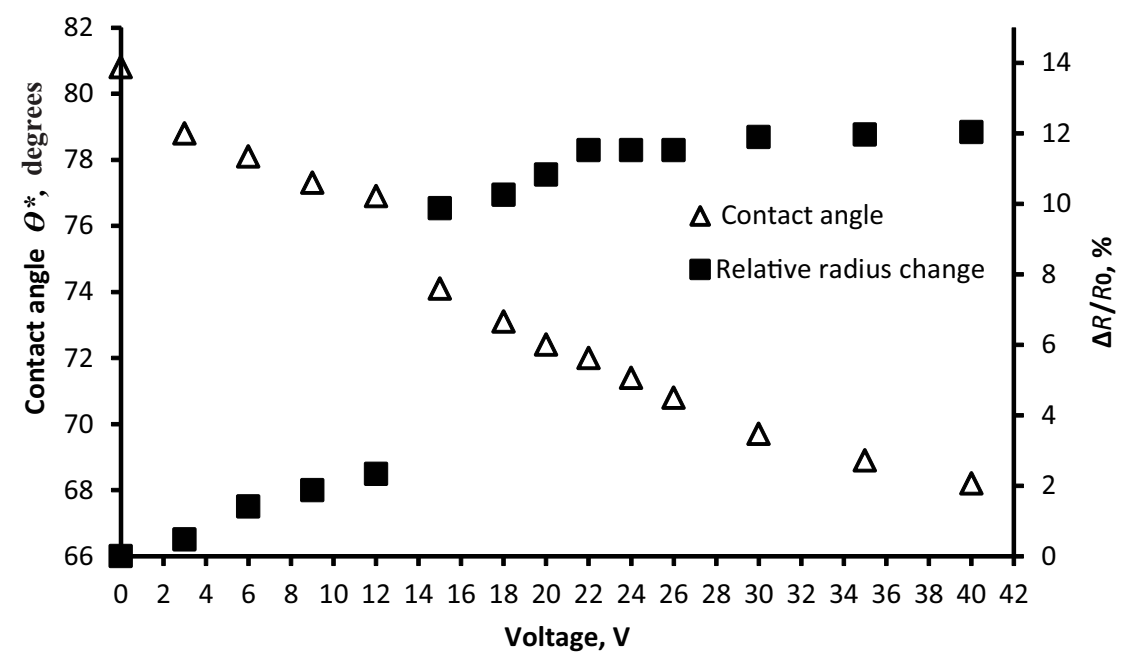

Figure $5 \mid$ The apparent contact angle $\theta^{*}$ and the dimensionless relative radius $\Delta R / R_{0}$ vs. applied voltage $U$ in the electrowetting experiment.

inherent for wetting transitions ${ }^{19-22,36-41}$. This value of voltage corresponding to the onset of wetting transition coincides well with the electrowetting voltage necessary for the Cassie-Wenzel transition reported recently in Ref. 42. The noticeable penetration of water through the membrane was observed at voltages as high as $1 \mathrm{kV}$. Such high values of the voltage make the use of membranes problematic.

In order to decrease voltage, we performed hydrophilization of PC membranes with the cold air radiofrequency plasma, as described in detail in the Methods Section. The plasma treatment creates a complex mixture of surface functionalities which influence physical and chemical properties of the surface. In particular, it results in a dramatic change of wetting behavior, resulting in the hydrophilization of the surface ${ }^{43-47}$. The initial apparent contact angle of plasma treated membranes was of $70-80^{\circ}$. It should be emphasized that despite hydrophilization they remained impermeable for water.

After the treatment of membranes with the cold air radiofrequency plasma we succeeded in obtaining water penetration by applying a relatively low voltage of dozens of volts, as shown in Fig. 4. It could be seen from Fig. 5 that a sharp decrease in the contact angle was accompanied by an increase in the contact line radius occurring at $12 \mathrm{~V}$. It is noteworthy that the decrease of the apparent contact angle, evidencing the wetting transition, was attended by the penetration of $5 \mu \mathrm{l}$ of water through the membrane. Deformation of the droplet was accompanied by detachment (de-pinning) of the triple (three-phase) line of the droplet. The displacement of the triple line established with goniometer is illustrated in Fig. 5. The precise microscopic picture of the water penetration remained unknown, but it is reasonable to suggest that de-pinning of the triple line led to the wetting transition ${ }^{19-22,36-41}$. We established that the maximal displacement of the triple line $\Delta R_{\max }$ equaled approximately $0.1 R_{0} \sim$ $100 \mu \mathrm{m}$, hence the de-pinned triple line slipped over and filled a dozen lines of pores, as estimated very roughly.

Non-contact electrical control of the permeability of membranes. A uniform electric field of $0-10 \mathrm{kV} / \mathrm{cm}$ was applied to the droplet, as shown in Fig. 1b. The electric field sufficient for deformation of the droplet was $2.5 \mathrm{kV} / \mathrm{cm}$ as shown in Fig. 6. Deformation of droplets by an electric field results from the complex interplay of electrical, surface tension and gravity-related effects ${ }^{48}$. The free energy $G$ of the droplet exposed to the electric field could be estimated as:

$$
G=G_{S}+G_{g r}+G_{e l}
$$

where $G_{S}, G_{g r}$ and $G_{e l}$ are surface, gravitational and electrical fieldrelated contributions to the free energy respectively, $G_{S} \cong \gamma S \cong$ $\gamma 4 \pi R^{2}, G_{e l} \cong \varepsilon_{0} \varepsilon E^{2} V / 2, G_{g r}=\rho V g h \cong \rho V g R / 2$, where $R, S, V$ and $h$ are the characteristic dimension, surface, volume and the height of the center mass of the droplet respectively; $\gamma, \rho$ and $\varepsilon$ are surface tension, density and dielectric constant of a liquid respectively ${ }^{48}$. The dimensionless constants $\xi$ and $\chi$ could be introduced as:

$$
\begin{gathered}
\xi=\frac{\varepsilon_{0} \varepsilon}{\rho g R} E^{2}, \\
\chi=\frac{\varepsilon_{0} \varepsilon R}{\gamma} E^{2} .
\end{gathered}
$$

a

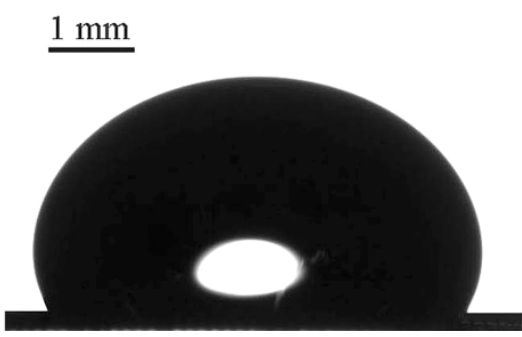

b

$1 \mathrm{~mm}$

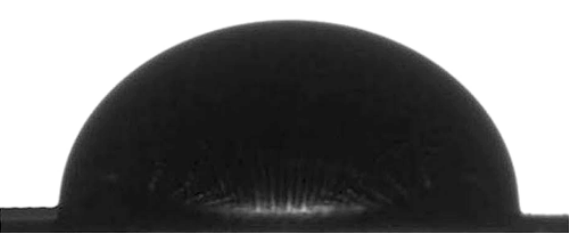

Figure 6 | (a) $40 \mu \mathrm{l}$ droplet of water/ethanol solution deposited on PC-coated gauzes. The obtuse apparent contact angle evidencing a pronounced Cassie-Baxter wetting is clearly seen. (b) The same droplet exposed to the uniform electric field of $2.5 \mathrm{kV} / \mathrm{cm}$. The abrupt change in the apparent contact angle evidencing a wetting transition is seen. 


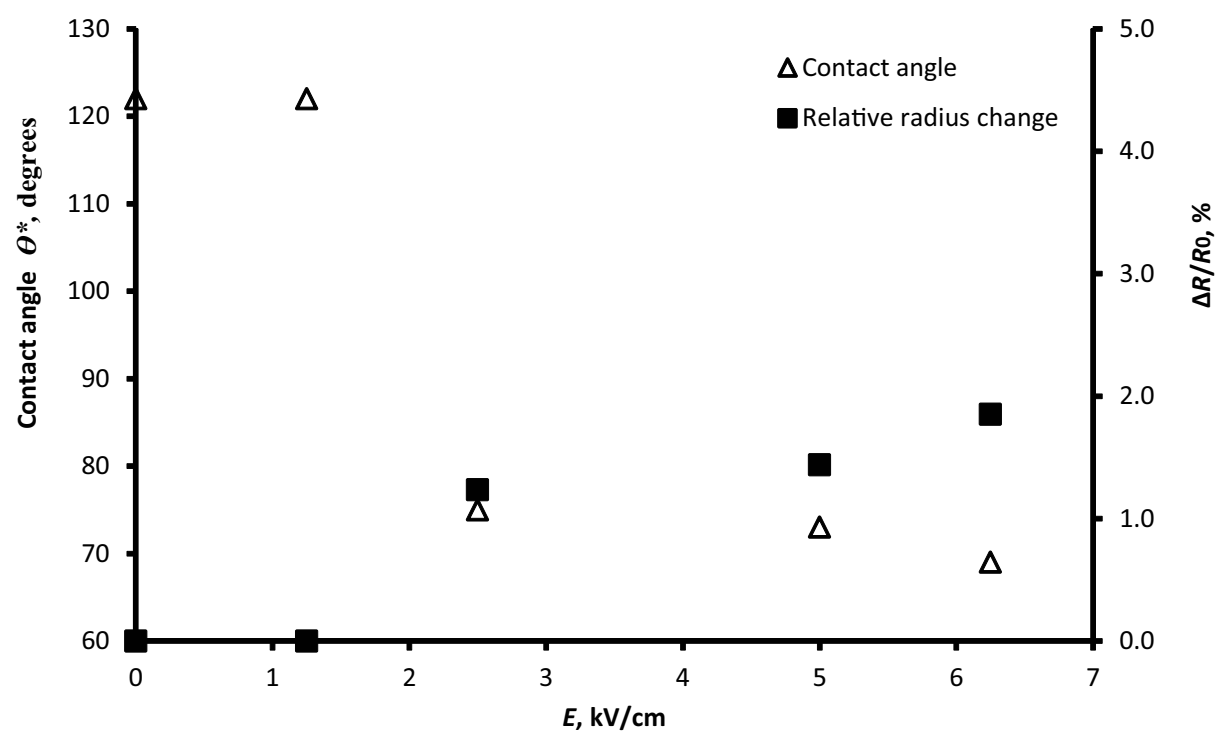

Figure $7 \mid$ The apparent contact angle $\theta^{*}$ and the dimensionless relative radius $\Delta R / R_{0}$ vs. applied electric field $E$ for the $20 \mu$ droplet exposed to the uniform electric field.

The dimensionless constant $\xi$ describes the interrelation of gravity and electrical field-induced effects, and the constant $\chi$ describes the interrelation of surface tension-induced and electrical effects. Substituting $R=1 \mathrm{~mm}, \rho=10^{3} \mathrm{~kg} / \mathrm{m}^{3}, \gamma=70 \cdot 10^{-3} \mathrm{~J} / \mathrm{m}^{2}, \varepsilon=80$ we obtain that when $E \sim 1 \mathrm{kV} / \mathrm{cm}, \xi \sim 1, \chi \sim 0.1$. It means that in our case the electrical energy is mainly spent as the counteraction to gravity.

The deformation of a droplet was accompanied by the de-pinning of the triple line and water penetration through the membrane, as shown in Fig. 7. However, the displacement of the triple line was much less pronounced when compared to the electrowetting experiment. The maximal displacement of the triple line $\Delta R_{\max }$ was equal to approximately $0.02 R_{0} \sim 20 \mu \mathrm{m}$, hence in this case the de-pinned triple line slipped over and filled only a couple of the lines of pores (recall that the average size of the pore was of $5 \mu \mathrm{m}$ ).

\section{Discussion}

The electrically controlled permeability of the membranes demonstrated in our study is based on the effect of the Cassie-Wenzel wetting transitions ${ }^{19-22,36-42}$. In our investigation wetting transitions were stimulated by exerting electric field on the liquid/porous solid system. One of the most debatable problems in the field of wetting is the problem of the "dimension" of the transitions, or, in other words: whether all pores underneath the droplet should be filled by liquid (the "2D scenario"), or perhaps only the pores adjacent to the threephase (triple) line are filled under external stimuli such as pressure, vibrations or impact (the "1D scenario") ${ }^{34,20,37-42}$. Our investigation does not supply an unambiguous answer to this question, but it demonstrates explicitly that the wetting transition is accompanied by a micro-metrically scaled displacement of the triple line depicted in Figs. 5, 7. It is plausible to suggest that the Cassie-Wenzel wetting transitions are mainly governed by wetting events occurring in the nearest vicinity of the triple line, as demonstrated in a series of recent investigations ${ }^{34,37-42}$.

We conclude that electrically driven membranes obtained with breath-figures self-assembly allow a low voltage $(\sim 10 \mathrm{~V})$ control of water penetration under conditions of electrowetting. The noncontact control of water penetration through membranes is also reported, however, it needs much higher voltage for the electrical deformation of a droplet. The permeability of membranes results from the Cassie-Wenzel wetting transitions, accompanied by the displacement of the triple line registered in both contact and non-contact schemes; however, the displacement of the triple line is more pronounced under conditions of the electrowetting experiment.

\section{Methods}

Membranes were manufactured with the fast dip-coating process. Stainless steel gauzes (depicted in Fig. 2) were used as carrying supports ${ }^{23}$. A 4 wt.\% PC solution was prepared by dissolving the polymer in a mixture of chlorinated solvents (chloroform, $\mathrm{CHCl}_{3}, 8$ wt.\% and dichloromethane $\mathrm{CH}_{2} \mathrm{Cl}_{2}, 88$ wt.\%, were supplied by BIO-LAB Ltd, both solvents were of the chemical grade purity). Thoroughly cleaned stainless steel gauzes were pulled vertically at a high speed of $v=40 \mathrm{~cm} \cdot \mathrm{min}^{-1}$ from the polymer solution and dried at room temperature and a $\mathrm{rH}$ of $50 \%$ in an environmental cell. Rapid evaporation of the solvent cooled the solution/humid air interface subsequently resulting in an intensive condensation of water droplets at the interface ${ }^{24-30}$. Water droplets then sank into the solution eventually forming microscaled pores typical for breath-figures self-assembly $y^{24-30}$. The topography of the membranes was studied with SEM (JEOL JSM 6510 LV, Japan).

Hydrophilization of membranes was achieved by exposure to an inductive cold air plasma discharge under the following parameters: the plasma frequency was on the order of $10 \mathrm{MHz}$, the power was $20 \mathrm{~W}$, the pressure was $P=6.7 \cdot 10^{-2} \mathrm{~Pa}$, the volume of the discharge chamber was $45 \mathrm{~cm}^{3}$. The time span of irradiation was $10 \mathrm{~s}$. After treatment, membranes were kept for 150 hours under ambient conditions $\left(24^{\circ} \mathrm{C}\right.$ and $50 \% \mathrm{rH}$ ) necessary for the hydrophobic recovery occurring after cold plasma treatment ${ }^{44-46}$. The apparent contact angle after completion of the hydrophobic recovery was approximately $90^{\circ}$ (mean of 10 measurements).

Distilled water $(\mathrm{pH}=6 ; \sigma=2.5 \mu \mathrm{S})$ was used for the electrowetting-driven water penetration study; a 98 wt.\% water/ethanol solution was used for the non-contact scheme. Ethanol (chemical grade, 96\% purity) was supplied by BioLab Ltd Israel.

Contact angles were established using a Ramé-Hart goniometer (model 500). Ten measurements were taken to calculate the mean apparent contact angle.

1. Eisenberg, S. R. \& Grodzinsky, A. J. Electrically modulated membrane permeability. J. Membrane Sci. 19, 173-194 (1984).

2. Grimshaw, P. E., Grodzinsky, A. J., Yarmush, M. L. \& Yarmush, D. M. Dynamic membranes for protein transport: chemical and electrical control. Chem. Eng. Sci. 44, 827-840 (1989).

3. Rios, G. M., Rakotodrisoa, H. \& Taroda de la Fuente, B. Basic transport mechanisms of ultrafiltration in the presence of an electric field. J. Membrane Sci. 38, 147-159 (1988).

4. Ly, Y. \& Cheng, Y.-L. Electrically-modulated variable permeability liquid crystalline polymeric membrane. J. Membrane Sci. 77, 99-112 (1993).

5. Golzio, M., Teissie, J. \& Rols, M.-P. Control by membrane order of voltageinduced permeabilization, loading and gene transfer in mammalian cells. Bioelectrochemistry 53, 25-34 (2000).

6. Kost, J. \& Langer, R. Responsive polymeric delivery systems. Adv. Drug Deliver. Rev. 64, 327-341 (2012).

7. Mugele, F. \& Baret, J.-Ch. Electrowetting: from basics to applications. J. Phys.: Condens. Matter 17, R705-R774 (2005).

8. de Gennes, P. G., Brochard-Wyart, F. \& Quéré, D. Capillarity and Wetting Phenomena. Springer, Berlin, 2003. 
9. Pollack, M. G., Fair, R. B. \& Shenderov, A. D. Electrowetting-based actuation of liquid droplets for microfluidic applications. Appl. Phys. Lett. 77, 1725-1726 (2000).

10. Quinn, A., Sedev, R. \& Ralston, J. Contact angle saturation in electrowetting. J. Phys. Chem. B 109, 6268-6275 (2005).

11. Bormashenko, E. Contact angles of sessile droplets deposited on rough and flat surfaces in the presence of external fields. Math. Model. Nat. Phenom. 7, 1-5 (2012).

12. Moon, I. \& Kim, J. Using EWOD (electrowetting-on-dielectric) actuation in a micro conveyor system. Sensor Actuat. A-Phys 130-131, 537-544 (2006).

13. Dai, W. \& Zhao, Ya.-Pu. The Nonlinear phenomena of thin Polydimethylsiloxane (PDMS) films in electrowetting. Int. J. Nonlin. Sci. Num. 8, 519-526 (2007).

14. Dai, W. \& Zhao, Ya.-Pu. An Electrowetting model for rough surfaces under low Voltage. J. Adhes. Sci. Technol. 22, 217-229 (2008).

15. Cho, S. K., Moon, H. \& Kim, Ch.-J. Creating, transporting cutting and merging liquid droplets by electrowetting-based actuation for digital microfluidic circuits. J. Micromech. S. 12, 70-80 (2003).

16. Taylor, G. Disintegration of water drops in an electric field. Proc. R. Soc. London Ser. A 280, 383-397 (1964).

17. Nayyar, N. K. \& Murty, G. S. The flattening of dielectric liquid drop in a uniform electric field. P. Natl. Sci. India A 25, 373-379 (1959).

18. Bormashenko, Ed., Pogreb, R., Stein, T., Whyman, G. \& Hakham-Itzhaq, M. Electrostatically driven droplets deposited on superhydrophobic surfaces. Appl. Phys. Lett. 95, 264102 (2009).

19. Patankar, N. A. Transition between superhydrophobic states on rough surfaces. Langmuir 20, 7097-7102 (2004).

20. Ishino, C., Okumura, K. \& Quere, D. Wetting transitions on rough surfaces. Europhys. Lett. 68, 419-425 (2004)

21. Nosonovsky, M. \& Bhushan, B. Patterned nonadhesive surfaces: superhydrophobicity and wetting regime transitions. Langmuir 24, 1525-1533 (2008).

22. Bormashenko, E. Wetting transitions on biomimetic surfaces. Phil. Trans. Royal Soc. A 365, 4695-4711 (2010).

23. Bormashenko, E., Balter, S., Bormashenko, Ye. \& Aurbach, D. Honeycomb structures obtained with breath figures self-assembly allows water/oil separation. Colloid Surface A 415, 394-398 (2012).

24. Widawski, G., Rawiso, B. \& Francois, B. Self-organized honeycomb morphology of star-polymer polystyrene films. Nature 369, 387-389 (1994).

25. Srinivasarao, M., Collings, D., Philips, A. \& Patel, S. Three-dimensionally ordered array of air bubbles in a polymer film. Science 292, 79-83 (2001).

26. Xue, L., Zhang, J. \& Han, Y. Phase separation induced ordered patterns in thin polymer blend films. Prog. Polym Sci. 37, 564-594 (2012).

27. Hernández-Guerrero, M. \& Stenzel, M. H. Honeycomb structured polymer films via breath figures. Polym. Chem. 3, 563-577 (2012).

28. Bormashenko, E., Balter, S. \& Aurbach, D. On the Nature of the breath figures selfassembly in evaporated polymer solutions: revisiting physical factors governing the patterning. Macromol. Chem. Phys. 213, 1742-1747 (2012).

29. Bormashenko, E., Pogreb, R., Stanevsky, O., Bormashenko, Ye., Socol, Ye. \& Gendelman, O. Self-assembled honeycomb polycarbonate films deposited on polymer piezoelectric substrates and their applications. Polym. Advan. Technol. 16, 299-304 (2005).

30. Liu, R., Liu, J. \& Liu, H. Fabrication of polycarbonate honeycomb porous films by Breath Figure method. Gaofenzi Cailiao Kexue Yu Gongcheng/Polymeric Materials Science and Engineering 29, 133-136 (2013).

31. Cassie, A. B. D. Contact angles. Discuss. Faraday Soc. 3, 11-16 (1948).

32. Cassie, A. B. D. \& Baxter, S. Wettability of porous surfaces. Trans. Faraday Soc. 40 546-551 (1944).

33. Nosonovsky, M. Multiscale roughness and stability of superhydrophobic biomimetic interfaces. Langmuir 23, 3157-3161 (2007).

34. Bormashenko, Ed. Wetting of real surfaces. De Gruyter, Berlin, 2013.

35. Tadmor, R. \& Yadav, P. S. As-placed contact angles for sessile drops. J. Colloid Interf. Sci. 317, 241-246 (2008).
36. Butt, H.-J., Semprebon, C., Papadopoulos, P., Vollmer, D., Brinkmann, M. \& Ciccotti, M. Design principles for superamphiphobic surfaces. Soft Matter 9, 418-428 (2013)

37. Bahadur, V. \& Garimella, S. V. Electrowetting-based control of droplet transition and morphology on artificially microstructured surfaces. Langmuir $\mathbf{2 4}$, 8338-8345 (2008)

38. Bahadur, V. \& Garimella, S. V. 1D Preventing the Cassie-Wenzel transition using surfaces with noncommunicating roughness elements. Langmuir 25, 4815-4820 (2009).

39. Bormashenko, E., Pogreb, R., Whyman, G. \& Erlich, M. Cassie-Wenzel wetting transition in vibrating drops deposited on rough surfaces: Is dynamic CassieWenzel wetting transition a 2D or 1D affair? Langmuir 23, 6501-6503 (2007).

40. Whyman, G. \& Bormashenko, E. Wetting transitions: general considerations. J. Adhes. Sci. Technol. 26, 207-220 (2012).

41. Papadopoulos, P., Mammen, L., Deng, X., Vollmer, D. \& Butt, H.-J. How superhydrophobicity breaks down. P. Natl. Acad. Sci. USA 110, 3254-3258 (2013).

42. Manukyan, G., Oh, J. M., van den Ende, D., Lammertink, R. G. H. \& Mugele, F. Electrical switching of wetting states on superhydrophobic surfaces: a route towards reversible Cassie-to-Wenzel transitions. Phys. Rev. Lett. 106, 014501 (2011).

43. Yasuda, H. Plasma for modification of polymers. J. Macromol. Sci. A 10, 383-420 (1976).

44. France, R. M. \& Short, R. D. Plasma treatment of polymers: the effects of energy transfer from an argon plasma on the surface chemistry of polystyrene, and polypropylene. A high-energy resolution X-ray photoelectron study. Langmuir 14, 4827-4835 (1998).

45. Kaminska, A., Kaczmarek, H. \& Kowalonek, J. The influence of side groups and polarity of polymers on the kind and effectiveness of their surface modification by air plasma action. Eur. Polym. J. 38, 1915-1919 (2002).

46. Bormashenko. Ed., Chaniel, G. \& Grynyov, R. Towards understanding hydrophobic recovery of plasma treated polymers: Storing in high polarity liquids suppresses hydrophobic recovery. App. Surf. Sci. 273, 549-553 (2013).

47. Mortazavi, M. \& Nosonovsky, M. A model for diffusion-driven hydrophobic recovery in plasma treated polymers. App. Surf. Sci. 258, 6876-6883 (2012).

48. Bormashenko, E., Pogreb, R., Balter, R., Gendelman, O. \& Aurbach, D. Composite non-stick droplets and their actuation with electric field. Appl. Phys. Lett. 100, 151601 (2012).

\section{Acknowledgements}

We are thankful to Mrs. N. Litvak for the high resolution SEM. We are thankful to Dr. G. Whyman for fruitful discussions. We are thankful to Mrs. Al. Musin for her kind help in preparing this manuscript.

\section{Author contributions}

E.B. analyzed data and proposed experimental concepts. R.P. planned and carried out experiments. S.B. planned and carried out experiments. D.A. analyzed data. All authors reviewed the manuscript.

\section{Additional information}

Competing financial interests: The authors declare no competing financial interests.

How to cite this article: Bormashenko, E., Pogreb, R., Balter, S. \& Aurbach, D. Electrically Controlled Membranes Exploiting Cassie-Wenzel Wetting Transitions. Sci. Rep. 3, 3028; DOI:10.1038/srep03028 (2013)

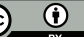

This work is licensed under a Creative Commons Attribution 3.0 Unported license. To view a copy of this license, visit http://creativecommons.org/licenses/by/3.0 